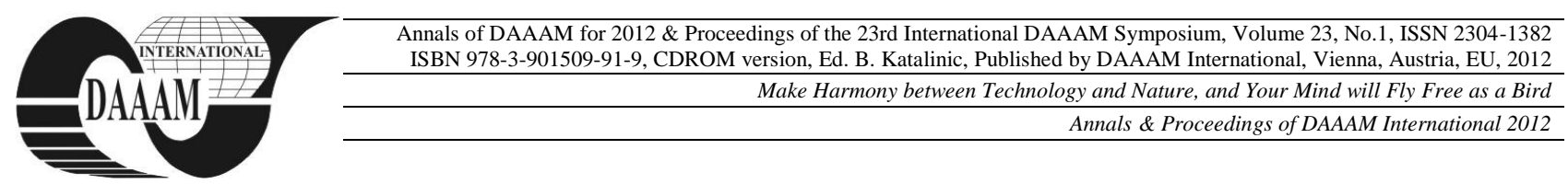

\title{
HOME AUTOMATION SYSTEM - AN EDUCATIONAL PROTOTYPE
}

\author{
DELAK, D[avid]; ZENZEROVIC, P[aolo] \& SUCIC, V[ictor]
}

\begin{abstract}
The main problem considered in this paper is household consumption monitoring and control. The proposed solution is basic, yet interesting because of its simple implementation. The proposed system enables control over household appliances (lights or virtually any kind of load) and monitoring of their power output. The proposed solution consists of a primary microcontroller (with its corresponding user interface) which communicates with several secondary microcontrollers arranged in a decentralized system. The system simultaneously sends power output consumption information to a PC, which gets analyzed and uploaded onto a server, thus enabling power consumption monitoring of the household wherever internet connection is available to the user.
\end{abstract}

Keywords: smart home, consumption monitoring, home control, domotics, microcontroller system

\section{INTRODUCTION}

The project described in this paper focuses on an important aspect of our everyday lives, which has reason to concern us even more in the future. That is energy consumption awareness [1]. Energy sources will, no doubt, change in the future to come: finding solutions to obtain electricity from renewable energy sources is becoming a priority even today [2]. However, the goal of this project is not to demonstrate reduction of energy consumption in one's home, but to make the user aware of his impact on the environment by putting onto scale his lifestyle's energy consumption. One possible way to do this is to enable some sort of home automation or home monitoring system. The term 'monitoring', in this case implies electrical power consumption monitoring. Although this function is not uncommon in home automated systems, monitoring is generally associated to the other meaning of the word 'monitoring' - that is to monitor and alarm if needed, hence to provide safety and security. Safety refers to the prevention of unwanted accidents such as: fire, flooding, gas leakage, etc.; while security refers to making the household secure from breaking in, by applying different technology such as digital locking systems (radio frequency identification, passwords, biometric scans, or other identification methods), video surveillance and sensors (glass-break, movement, thermal and other sensors) [3]. The system described in this paper is an example of a do-it-yourself solution. The system has a simple design and programming, it offers few functions and settings, yet it is personalized for the user's needs.

\section{EXISTING SOLUTIONS}

There is a great number of existing solutions on the market regarding home automation and monitoring, both professional and non-professional [4,5]. Each of those systems has its advantages and drawbacks. One of the most interesting properties however is a good functionality/cost ratio. From a real estate point of view, home automation increases the value of the household, which shall be even more accented in the future.

\subsection{Professional solutions}

Numerous professional solutions combine hardware modules with PC software in order to maximize control over the household and improve flexibility and ease of use as well. The variety of available systems offers a great deal of possibilities which varies the system price greatly, therefore it is important to study the system's possibilities and features before choosing a system to install. Compatibility with other manufacturers' hardware often is a deciding point for buyers [6]. The new generation of home automation focuses on greater user mobility, thus enabling control and monitoring options via mobile devices. What is common in most professional solutions today is: loads switching (on/off), temperature monitoring, occupancy sensors, irrigation control, hi-fi media center implementation, home security, fire/flood alarm, light scenes, video surveillance, events logging, consumption analysis and charting etc.

\subsection{Low-cost solutions}

Low-cost solutions besides being affordable require some know-how. That is mainly because the user buys the needed components and assembles them individually. There are various communities and websites which offer free information on microcontrollers and home automation, some even provide basic project layouts which can be modified. An interesting solution consists of an ethernet enabled Arduino board combined with an Android mobile device [7]. A wireless modem/router is required to enable control over the board with a mobile phone. The functionality is basic, concerned with switching user-defined loads and monitoring temperature.

Almost all low-cost solutions focus on: loads switching (on/off), temperature monitoring, security. More advanced projects share some professional features like: IP video surveillance, control via mobile devices, consumption charting, etc. Since the user assembles and programs the hardware, the results are greatly influenced by the user knowledge and expertise. 


\section{IMPLEMENTED SOLUTION}

The solution described in this paper addresses two main features: load control and total power consumption readout. The implemented solution is not intended for industrial usage but is offered as an open hardware and open software alternative. Also, the implemented system is proposed as an educational technology tool for electronics engineering studies [8]. The block diagram of the proposed system is shown in figure 1 .

The system is composed out of one master module and multiple slave modules. A simulation has been run with one master microcontroller, and two slave microcontrollers. The master microcontroller makes it possible to control every load in the household, while the slave microcontrollers follow commands issued from the master.

The master module has navigation buttons and an alphanumeric LCD installed to act as a human-machine interface. The idea is to install the master module in that part of the household where it is most needed or where inhabitants spend most of their time (e.g. the living room). From that central point the user can control all other slave modules, the number and distribution of which depends solely on the user.

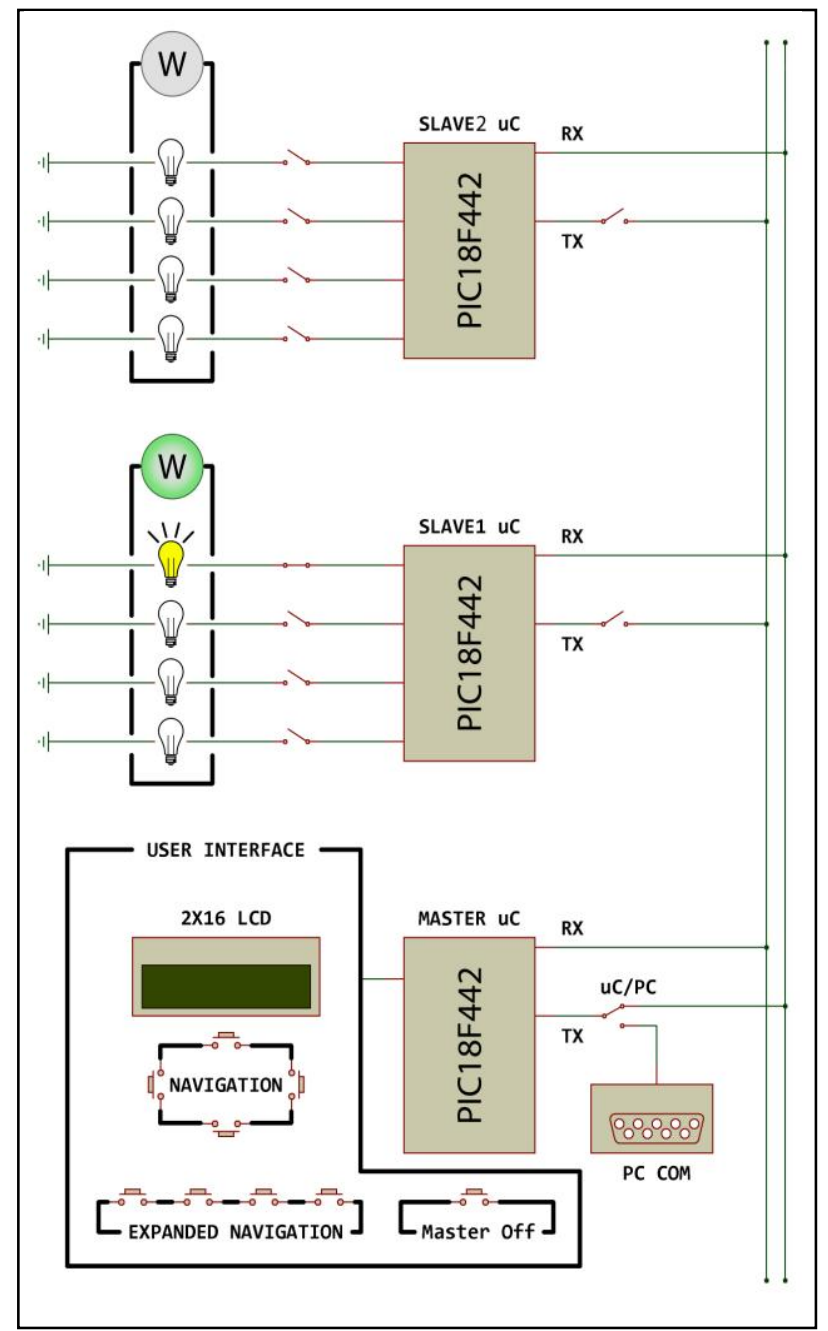

Fig. 1. Project block diagram
Every slave module has the capability of controlling four loads, as shown in figure 1. If needed, multiple slave modules could be installed in a single room, depending on the number of loads the user needs control over. The number of slave modules can be increased in real life, depending on the application. The only limiting factors to the number of slave devices are the communication protocol and the slave identification number. Since both UART communication protocol and slave ID number assignation method support 8-bit binary numbers, those factors limit the number to 255 slaves in a single system.

\subsection{Functionality}

The master module functionality enables menu navigation (user interface), programming slave modules, controlling the state of loads attached to slave modules, total output power display, and communication with the personal computer.

The slave module functionality enables the control of four loads per slave (switching on/off) via buttons or switches connected to the slave or via a remote link. Also, the slave enables four current sensing pins for power monitoring purposes. The master modules can send queries to the slave module to receive all the values of the observed variables.

The master module menu has been organized in such a way that it provides quick access to all functions while retaining simplicity. Special care was taken when programming the menu and submenus to keep an intuitive disposition of its contents; so first time users could get by quickly. The menu structure is illustrated below, listing main entries only (without their submenus):

\subsection{Main Menu \\ 1. Control \\ 2. Power \\ 3. Program}

The 'Control' submenu is accountable for controlling slave module load states (on/off). A 'Master Off' button is implemented in the design to instantly turn off all loads at any time. This is a practical solution when leaving the household in a hurry or if safety threat occurs.

The 'Power' submenu enables real-time displaying of electrical power consumption with a refresh interval of one second.

The 'Program' submenu gives the user the possibility to associate each slave module button-load arrangement in a configuration different from the default one (that is: button1-load1, button2-load2, etc.). Assigning multiple loads to one button, and multiple buttons to one load is possible, allowing more flexibility depending on the arrangement of buttons in the room or desired adaptability. 


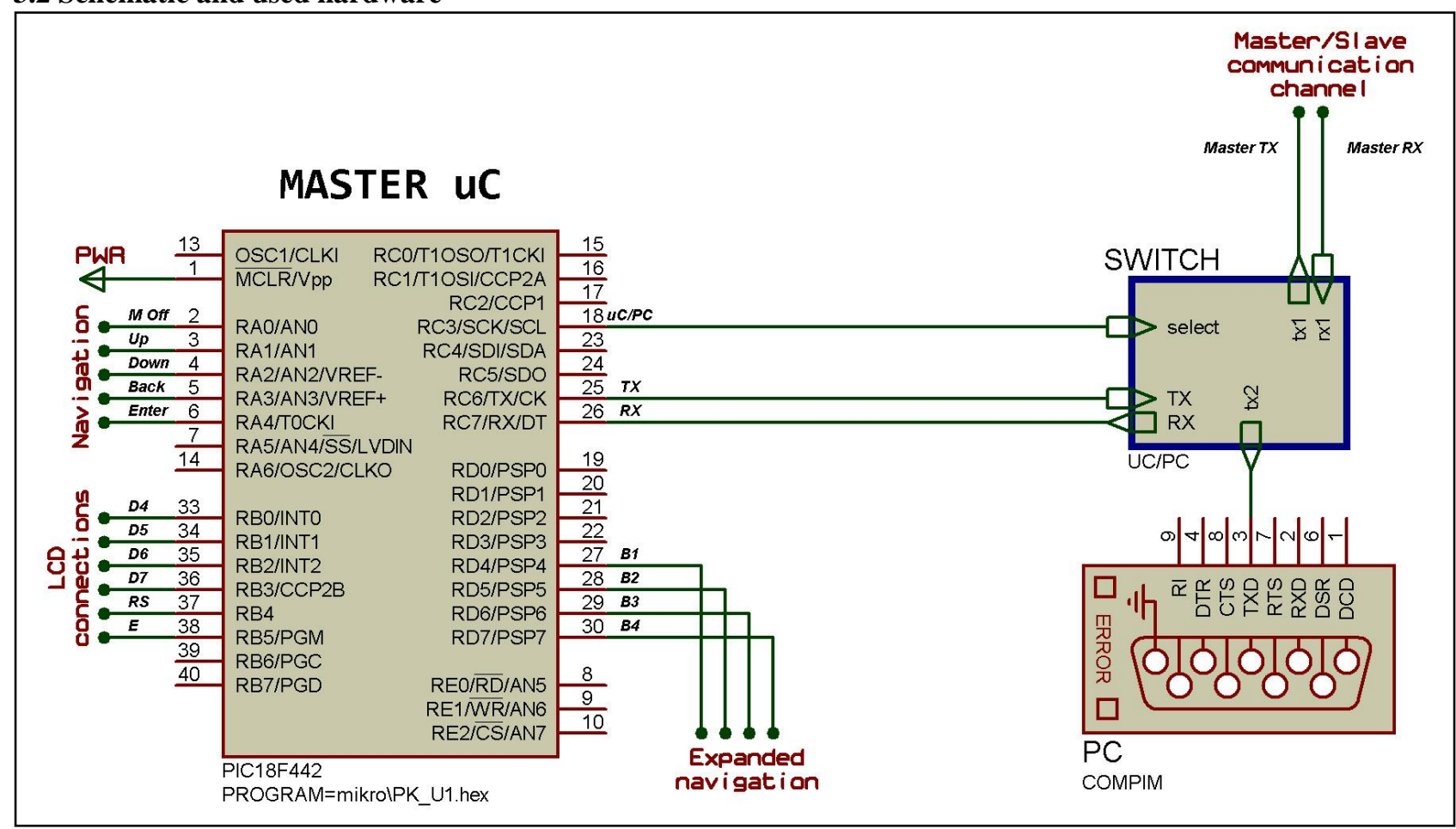

Fig. 2. Master microcontroller peripherals

The hardware interaction was simulated with the aid of the simulation package 'ISIS Proteus7 Professional', while the program code for master and slave modules was developed and compiled in the programming suite 'Mikroelektronika mikro Pascal PRO for PIC'. The complete system with its hardware and software is developed as an open source project. The most recent data and documentation about this system can be found at: www.cset.com.hr/house-power-meter.htm

The hardware layout of the master module is shown in figure 2. The slave module hardware layout is shown in figure 4.

The user interface associated to the master module consists of a two lined alphanumeric LCD display and four navigation buttons ('Up', 'Down', 'Left' and 'Right').

Four more buttons ('B1', 'B2', 'B3' and 'B4') may be thought of as part of an expanded navigation which helps the user to easily setup button-load configurations in 'Program' mode, and to faster change load states in 'Control' mode. Those additional buttons are not to be confused with the ones that are part of each slave module - in spite of the same designation -yet should be thought of as an addition to the navigation of the main module which enables a more straightforward approach in regards of control and setup of slave modules.

An additional 'Master Off' button is designed to quickly turn off all loads in the system. The master module needs to communicate with all slave modules in the system, as well as with a personal computer to enable network monitoring of the household's power consumption.
Because data needs to be sent and received from master to slave modules, a two-way communication is required; however the communication with the personal computer is unidirectional, from the master module to the personal computer (because no data needs to be received from a personal computer in this project). A subcircuit shown in figure 3 composed of digital switches and logical gates is responsible for switching the connection between master module and slave modules or personal computer communication. This is a simple solution for establishing two connections via one UART module, although a better alternative would be choosing a different microcontroller as a master unit, which features two independent UART modules.

All slave modules, with their immediate components, share the same hardware schematic. They are composed of four buttons - which (by default) control respectively four loads - and four simulated current probes (each measuring one load's current). Having the same hardware layout and programming, slave modules achieve their modular characteristic.

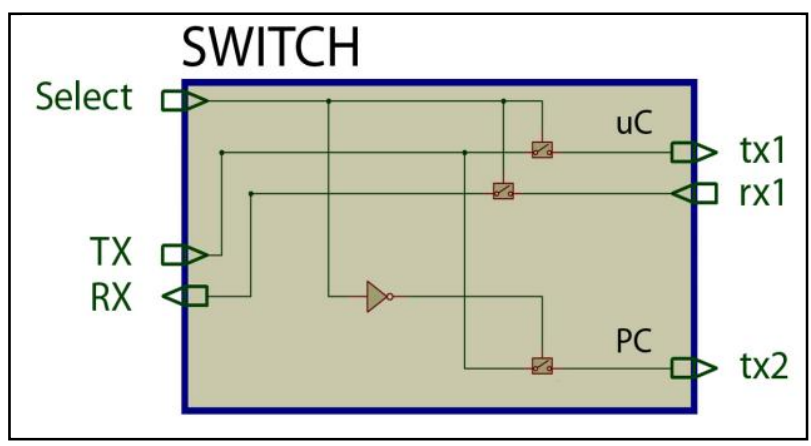

Fig. 3. Master microcontroller UART subcircuit switch 


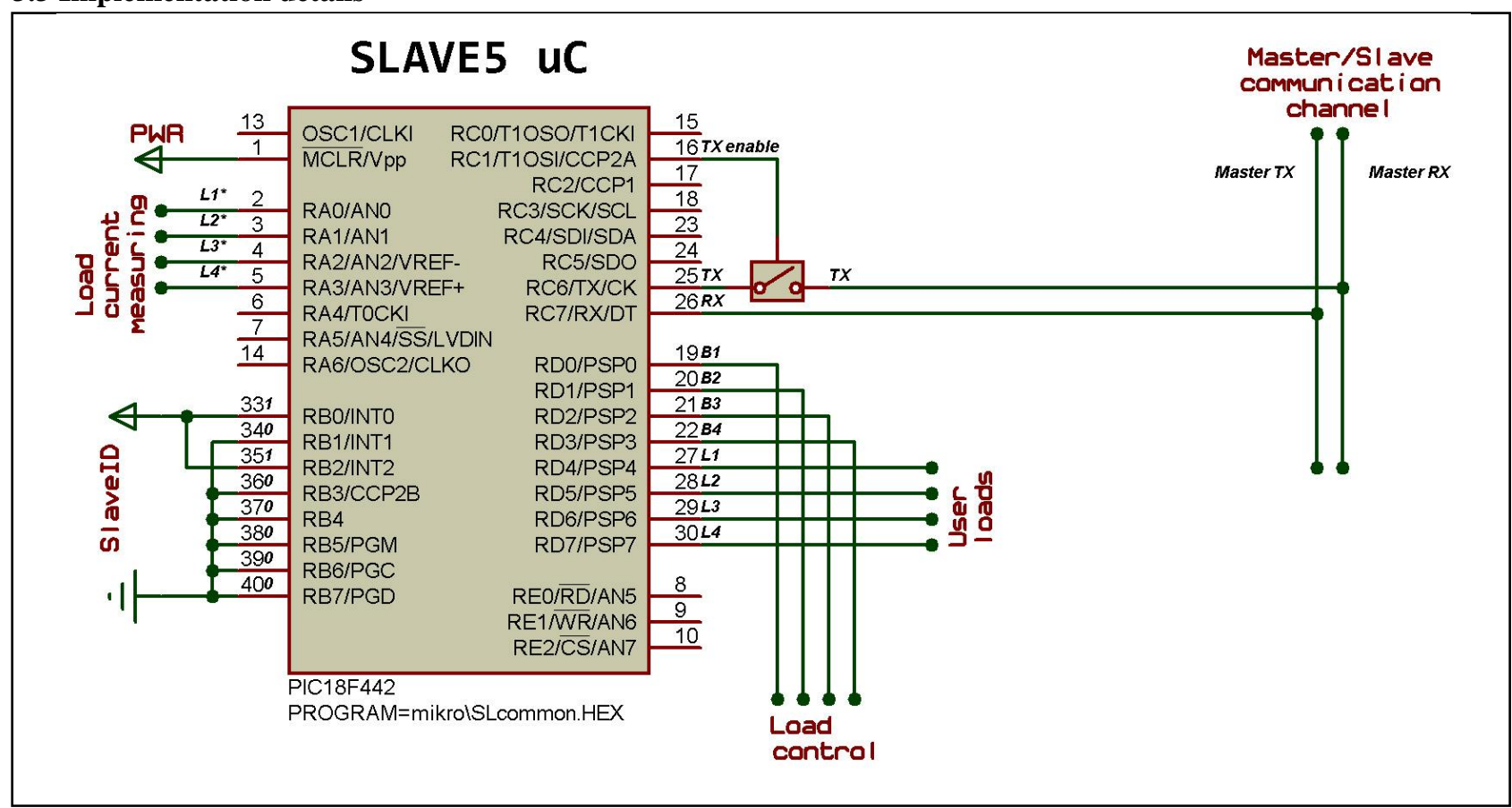

Fig. 4. Slave microcontroller peripherals

Slave modules are identified by their 'slave ID' number. The slave modules are identical both in the hardware and software. The slave ID number is assigned physically by grounding or connecting to the power terminal the pins on PORTB using dip switches: this resembles a binary number of eight bits which corresponds to the slave ID number.

This method is very simple and makes manual 'reprogramming' of the slave modules possible at any time without modification to the module's software.

For all the communication of master and slave modules a serial protocol has been chosen. Using the serial UART protocol is tricky in case of multiple device communication, because of the logic contentions that take place on a common communication channel. In this case logic contentions would take place on the master module's receive (RX) pin due to the fact one of the slave modules could be transmitting a message (byte) while all the others are idle. As seen on schematic, this problem is solved by adding digital switches on all slave modules' transmit (TX) pin. Logic contentions do not take place on the master module's TX pin, so there's no need for switches there. In this manner all slave modules receive the same message, but only one of them turns on its switch, thus preventing logic contentions, and establishes a two-way communication with the master module.

Essentially the master-slave communication shares a resemblance with how people communicate. To successfully send a message to any slave module, the master module needs firstly to address the correct one this is done by sending the first byte through the communication channel, which is always the slave ID number (except when pressing the 'Master Off' button).
Next, in order to tell the slave module what to do, two more bytes are sent, because an unambiguous message cannot be sent with one byte at this point. The second byte to be sent through the communication channel sets the chosen slave module in the correct program loop, which basically means it tells it what to expect as a third byte (except for 'Power' mode, load status queries and button configuration queries - in those cases a third byte will not be sent). The second byte sent by the master module will be referred to as a 'command ID' byte.

The master-slave communication is conceptualized as follows:

1. master module sends slave ID (e.g. ' 1 ')

2. respective slave module (e.g. Slave1) waits for command, other modules ignore communication

3. master module sends command ID (e.g. ' 100 ' for Control mode)

4. slave module enters respective program loop

5. master module sends command byte (e.g. '1100xxxx')

6. slave module executes command (e.g. set loads L4 and L3 on, L2 and L1 off)

Table 1 shows the command ID bytes and their meaning in the communication protocol:

\begin{tabular}{|c|c|}
\hline \multicolumn{2}{|c|}{ Command ID } \\
\hline Code & Description \\
\hline 100 & Control mode \\
\hline 101 & Power mode \\
\hline 102 & Program mode \\
\hline 110 & get load status \\
\hline 111 & get button configuration \\
\hline 255 & turn off all loads \\
\hline
\end{tabular}

Tab. 1. Master-slave 'command ID' bytes 
When 'Control' mode is selected, the master module addresses the desired slave module, sends a code 110 and waits for a response. When a slave module receives a code 110 , it sends to the master module a byte that is essentially a representation of the all the loads statuses. The master module displays the statuses on the LCD as a four-digit binary number - each digit representing one slave module load status ( $0=$ 'off', $1=$ 'on').During 'Control' mode the master module inquires the slave modules for loads status, thus making possible displaying momentarily any change that takes place remotely (if slave module buttons are pressed). While slave module loads status changes are checked periodically, a control signal (code 100) with its corresponding message is sent only if master module buttons (B1 - B4) are pressed. In that case the slave module sets its outputs to match the received message.

When in 'Program' mode, the master module addresses the desired slave module and checks its button configuration (code 111). Editing this configuration is then possible and is finished when 'Enter' button is pressed: the master module addresses again the desired slave module and issues a program signal (code 102) with its corresponding message. The byte containing slave modules button configuration information is to be analyzed two bits at a time. Having eight bits, a byte is suitable for transmitting all required information to the slave module: two bits form four combinations (four buttons) while their placement inside of the byte also forms four combinations (four loads). Using a similar approach slave modules have been programmed to store in their EEPROM memory their button configuration depending on the memory address $(1-4)$ : the address defines the button while the content defines the load.

This concept is illustrated in figure 5, and enables the dynamic allocation of load switching and buttons usage.

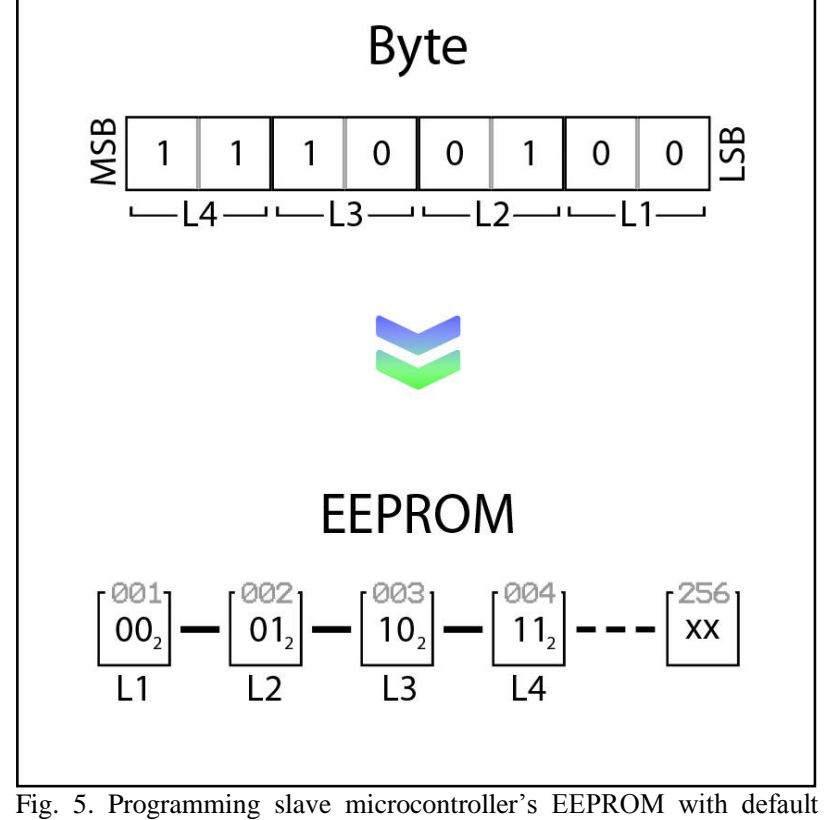

load-button configuration
Code 255 is sent to all slave modules whenever the 'Master Off' button is pressed, and instantly turns off all loads. Since all loads need to be turned off, addressing all slave modules one at a time would take too much time in a configuration with a great number of slave modules; therefore it is required to address them all simultaneously, and that is the reason why this design has a virtual limit of 255 slave modules: slave ID numbers may vary from 0-254 (which makes a total of 255 possible slave module identification numbers), while ' 255 ' cannot be a slave ID number, for it is a reserved code for the 'Master Off' feature. For any average user this limitation on the number of slave modules installed in the system surely would not represent a drawback of the design. Perhaps an industrial application would require a much bigger number of slave modules, and an even greater number for a system limit. However this design was never intended for professional/industrial usage, yet personal - and as such proves to be fit.

The system is projected to carry out power consumption readouts. Four current probes are connected to each slave module, giving a reading from 0-5 Volts. This analog voltage is converted into a 10-bit digital value, giving a resolution of 1024 steps. A 10-bit analog to digital converter gives small conversion errors, allowing fairly accurate power readouts. Supposing a maximum measurable value of 20 Amperes per load, and having a constant voltage of 220 Volts, power measuring errors due to ADC are expected to be less than 5 Watts. The final resulting error might reach a greater value due to stochastic errors and the fact that all hardware does not have ideal characteristics. In some branches of industry a 5 Watt measuring error might be of crucial significance, as in others would be negligible. Altogether, power measuring may be considered as fairly accurate in view of the project application.

\subsection{Advantages and disadvantages}

Both the advantage and disadvantage of this solution are its simplicity. This is an advantage for inexpert users who want a low-cost solution with basic features. On the other hand, advanced users will consider professional solutions or create a solution of their own to suit their needs.

The system advantages are:

- simple design (low number of components)

- quick setup and modularity (easily expandable)

- low cost

- upgradeable design and program (increasable functionality and quality)

The disadvantages are:

- basic functionality

- communication protocol

- system failure in case of master module failure 


\section{NETWORK MONITORING SOLUTION}

The power output readouts are sent to a PC for further analyses. A serial COM port is used to establish a connection between master module and personal computer. By implementing a network monitoring solution, the system not only can display power consumption information via the user interface, but also via an internet browser. This way, viewing power consumption on a graph in real time is possible from practically anywhere.

\subsection{Functionality}

The system has a one-second check interval of all loads power consumption. It is a cumulative, not average value, expressed in kilowatts. Read data is sent via UART to a COM port.

\subsection{Implementation details}

Since the system is simulated, a pair of virtual COM ports has been created. Data is sent from the hardware simulation software to one of the virtual ports, and as a consequence is received by the second virtual port which is paired. Using Java programming language the second COM port is defined as an input for collecting power consumption data sent from the master module. It is essential to setup properly COM communication by matching various settings (port name, baud rate, data bits, stop bits, parity) in the hardware simulation software, serial port simulation software and Java code.

The readouts are uploaded to a server so they can be accessed by the user anytime, from anywhere. In order to realize this, a virtual server is simulated as well. "Apache Friends XAMPP" software has been used to create a virtual Apache web server. Once again Java is used to establish a connection with the server through a socket and to send URLencoded information. In order to establish a connection through a socket some additional information is required, such as: host name, inet address and port. Data is stored by Java and is accessed using PHP and HTML programming. In order to represent a realtime graph in a browser, Ajax and Javascript programming languages are implemented.

Data can now be visualized on a graph in realtime, as shown in figure 6.

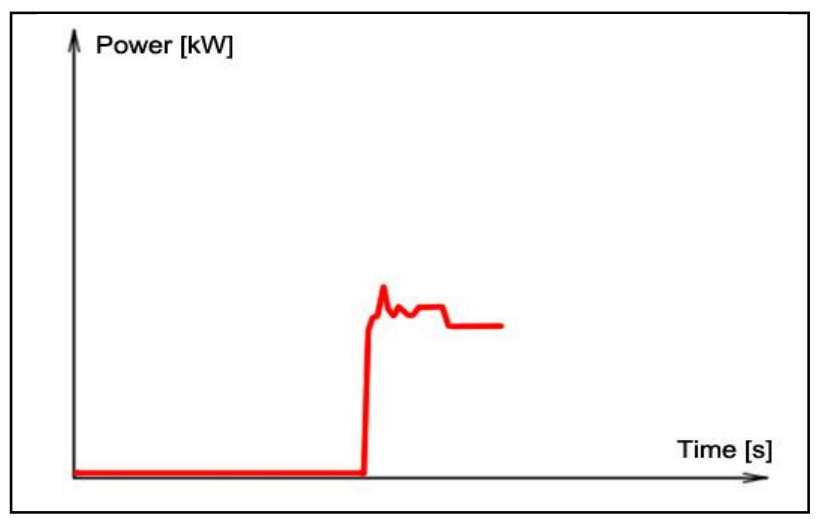

Fig. 6. Realtime consumption visualization

\subsection{Advantages and disadvantages}

There are both advantages and disadvantages for this type of network monitoring solution. One hardwarebased disadvantage will be mentioned in this section, for it concerns the network monitoring solution.

The advantages are:

- functional design with room for improvement

- data logging and charting

- access from anywhere in the world

The disadvantages are:

- various programming languages

- multiple interweaved technologies (Java, PHP, MySQL, Javascript)

- physical UART switching from module to PC

\section{CONCLUSION AND FUTURE WORK}

The proposed solution is simple, but novice users would probably be satisfied with its functionality. Fortunately, microcontrollers find use in a wide number of applications because of their reliability and possibilities; so improvements of this design, both from the hardware and programming point of view, are possible. What would seem logic as a next step for this project, would be to improve existing functions and to offer new ones, to maximize user-friendliness, and tackle problems related with the mentioned disadvantages of the design. Since hardware communication is of crucial significance, a new communication method should be implemented in such a way to minimize the possibility of system failure due to hardware malfunction or hacking. This means creating a self-aware system which can adapt to minor layout changes.

\section{ACKNOWLEDGEMENTS}

The author wants to express special thanks to the Faculty of Engineering, University of Rijeka and the Croatian Society for Educational Technology for its support in this project.

\section{REFERENCES}

[1] Abdelmohsen, S. \& Yi-Luen Do, E. (2008). Energy Puppet: An Ambient Awareness Interface for Home Energy Consumption, Available from:

https://wiki.cc.gatech.edu/designcomp/images/3/38/SID-EnergyPuppet.pdf Accessed on: 2012-09-19

[2] http://www.greenchoices.org/green-living/energy/conventional, (2009). Accessed on: 2012-09-04

[3] Stavridou, V.; Dutertre, B. (1998). From Security to Safety and back, Proceedings of Computer Security, Dependability and Assurance: from Needs to Solutions, York, UK, 0-7695-0337-3, pp. 182-195

[4] http://www.homeauto.com/main.asp , Accessed on:2012-09-04

[5] http://www.homeautomationindex.com , Accessed on: 2012-09. 04

[6] http://hometoys.com/emagazine.php?url=/ezine/11.10/calderone 5/index.htm , (2011). Accessed on: 2012-09-04

[7] http://www.domotichome.net, Accessed on: 2012-09-04

[8] Ringstaff, C. \& Kelley, L. (2002). The Learning Return On Our Educational Technology Investment, Available from: http://dmps.typepad.com/tac/learning_return.pdf Accessed on: 2012-09-19 